\title{
Hereditary hemorrhagic telangiectasia: an under-recognized but potentially serious condition
}

\author{
Morgan MacDonald BKin', Emad Massoud MD² \\ 'Class of 2017, Faculty of Medicine, Dalhousie University \\ ${ }^{2}$ Division of Otolaryngology, Department of Surgery, Dalhousie University
}

\begin{abstract}
Hereditary hemorrhagic telangiectasia (HHT) is a vascular condition that is inherited in an autosomal dominant fashion and demonstrates variable epidemiology by region. Delays in diagnosis are common, due to the low frequency of HHT, which may lead to serious complications and poor health outcomes. The Curaçao criteria should be used to assist with the diagnosis of HHT and to identify patients who require further investigations. This report provides an example of a patient whose diagnosis of HHT was delayed, and how the appropriate investigations were completed. We also give a brief summary of the complications of HHT and available treatments options.
\end{abstract}

42-year-old female presented with a one-week Ahistory of recurrent painless bleeding from her tongue with eating, each episode lasting approximately one hour. The patient also reported a prolonged history of recurrent nosebleeds.

Her past medical history was significant for hypothyroidism and chronic anemia. These were treated with levothyroxine and iron supplements, respectively. She had not been diagnosed with any other comorbidities and was not taking any other medications at the time. The patient reported that her sister also had nosebleeds and skin lesions similar to her own.

On examination there were blanchable, small vascular lesions along the lips, fingertips, oral cavity, nasal septum, dorsum of the tongue, and upper chest. Due to the history of recurrent nosebleeds, and telangiectases in characteristic locations, a diagnosis of hereditary hemorrhagic telangiectasia (HHT) was entertained and the patient was sent for a computed tomography (CT) of the chest and magnetic resonance imaging (MRI) of the brain. The chest CT demonstrated a lesion in the right lower lobe suggestive of a small arteriovenous malformation (AVM). With this additional information a diagnosis of HHT was made.

Bleeding from the tongue lesions resolved spontaneously once counseled to apply direct pressure to the bleeding area however, her epistaxis eventually required potassium titanyl phosphate (KTP) laser therapy, which was well tolerated. A brain MRI did not show any evidence of cerebral venous malformations (CVMs) and gastrointestinal imaging, including Barium swallow and small bowel follow through, demonstrated no abnormalities. Serial chest CT imaging and pulmonary perfusion studies continued to show a small stable right lower lobe AVM.

\section{Discussion}

HHT, also known as Osler-Weber-Rendu syndrome, is a vascular condition that is inherited in an autosomal dominant fashion with more than 600 different HHT-causing mutations. ${ }^{1-3}$ The prevalence of HHT is variable among different ethnic groups and is difficult to estimate because of frequent asymptomatic presentations. Rates as high as 1:2,350 have been reported in certain regions of France and 1:3,500 on the Danish island of Funen. ${ }^{4}$ Rates of HHT in Europe and North America are generally reported to be in the range of 1:10,000 but rates as low as 1:39,000 have also been identified in Northern England.,5

Given the low frequency of HHT, many physicians do not appreciate the range of clinical manifestations it may have. This often leads to misdiagnosis or delays in diagnosis. ${ }^{6}$ The morbidity and mortality caused by HHT can be severe, which is why considering HHT in a patient with unexplained telangiectasias or recurrent spontaneous bleeding, particularly in the form of epistaxis, is important. ${ }^{7}$

\section{Criteria}

1. Spontaneous epitaxis

2. Multiple telangiectases in characteristic sites

3. Visceral arteriovenous malformation

4. A first-degree family member affected with HHT

Diagnosis

Definite diagnosis: 3 criteria are present

Possible diagnosis: 2 criteria are present

Unlikely diagnosis: 1 criterion is present

Table 1. The Curaçao Criteria. 

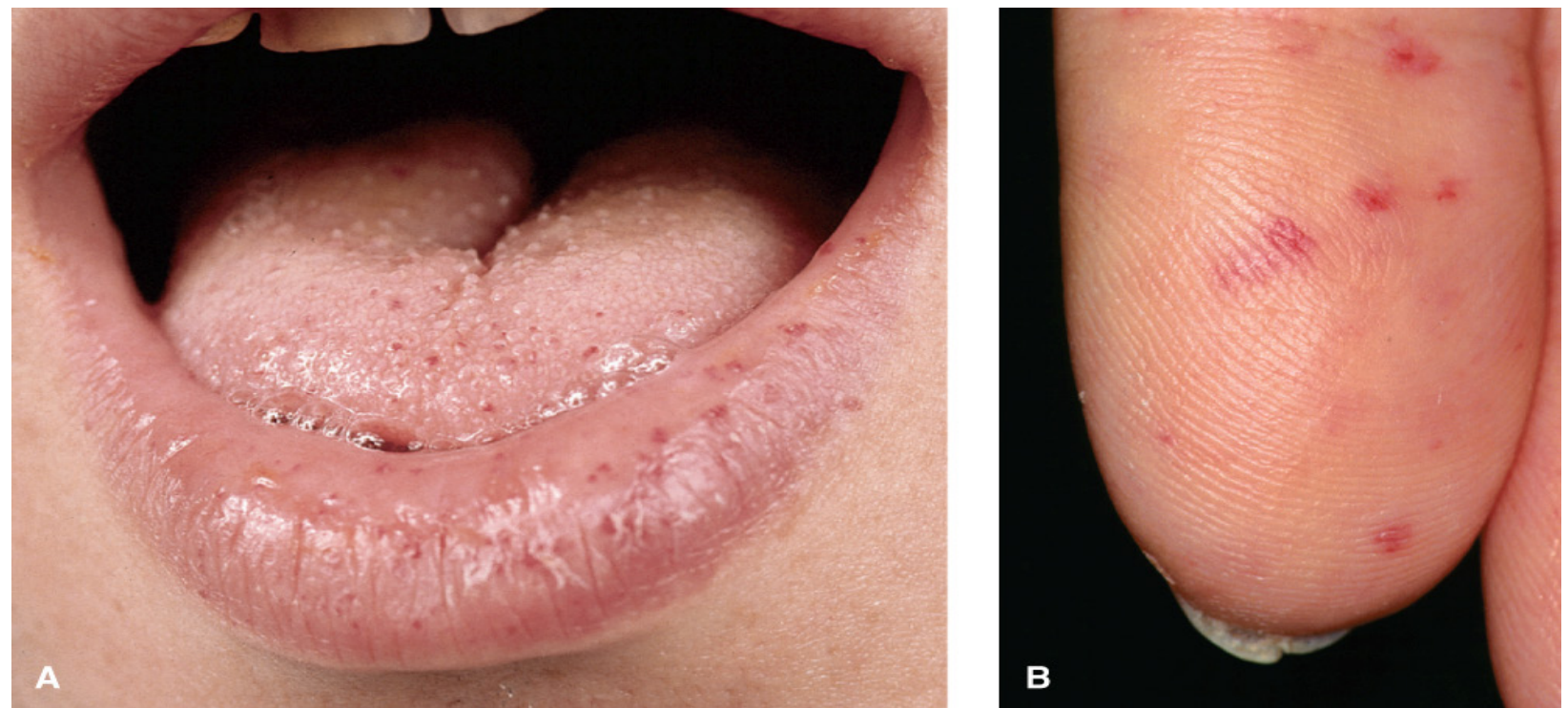

Figure 1. Telangiectasias on oral mucosa (A) and fingers (B). Credit to: Wolff K, Johnson R, Saaverda A. Ch.16 Genetic Diseases. In Fitzpatrick's Color Atlas and Synopsis of Clinical Dermatology, Seventh Edition. 2009.

The Curaçao criteria have been used to help make a clinical diagnosis of HHT (Table 1). ${ }^{5}$ These criteria look at four factors: recurrent spontaneous epistaxis, mucocutaneous telangiectases at characteristic sites including fingers, lower lip, buccal mucosa, and tongue (Figure 1), a visceral arteriovenous malformation, and a first-degree family member affected with HHT. An individual must have at least three criteria to be diagnosed with HHT. If only two of the criteria are present, then HHT is considered possible. HHT is considered unlikely if only one of the criterion is present. HHT demonstrates incomplete penetrance and when it does present, symptoms vary in severity. Some individuals may present with severe recurrent gastrointestinal bleeding requiring frequent blood transfusions, while others may present with mild episodes of epistaxis. ${ }^{4}$ Due to these confounding factors, a clear clinical diagnosis can be difficult and genetic sequencing may be needed to aid with the diagnosis. ${ }^{5}$ Approximately $87 \%$ of patients with HHT will demonstrate a mutation in the genes ENG or ACVRL commonly found on chromosomes 9 and 12 . There are still $10 \%$ of the individuals who meet the diagnostic criteria for HHT who will not have any

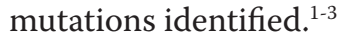

Bleeding caused by HHT can occur anywhere in or on the body but is more frequent in specific regions of the body and rarely occurs on the skin. The most common symptom of HHT is epistaxis, which occurs in $95 \%$ of affected individuals. Other areas commonly affected by bleeding due to HHT are the lungs, the central nervous system, as well as the digestive system including the alimentary tract, liver, and pancreas. ${ }^{4}$
Treatment of bleeding in the alimentary canal is dependent on the severity. Cauterization and photocoagulation may be used if blood loss is significant, however many patients can be treated with iron supplementation and blood transfusions as needed. Hepatic bleeding is usually asymptomatic and if it does become symptomatic, liver transplantation may be required. ${ }^{8}$ Screening for gastrointestinal bleeding in these patients should include hematocrit, hemoglobin, and iron studies starting at the age of 35. Endoscopic studies are only needed if anemia is present and disproportionate to epistaxis.,10

The likelihood a patient will have pulmonary symptoms largely depends on the specific gene for the condition that is inherited by the patient. Approximately $40 \%$ of individuals with HHT will develop pulmonary arteriovenous malformations (PAVM). ${ }^{9}$ It is also estimated that $60 \%$ of patients with pulmonary arteriovenous malformations have HHT. ${ }^{1}$ Common pulmonary symptoms for HHT are dyspnea, fatigue, and cyanosis. Interestingly, patients may present with secondary neurological problems such as brain abscess, transient ischemic attack and ischemic stroke without pulmonary symptoms. These neurologic problems occurs exclusively in patients with pulmonary arteriovenous malformations. ${ }^{4}$ Treatment for pulmonary symptoms of HHT may include resection of the affected lung, ligation therapy and transcatheter embolotherapy of the affected area. ${ }^{8}$

Neurological complications caused by PAVMs are a significant source of morbidity and mortality in patients with HHT, warranting screening at the time of diagnosis regardless of age. Transthoracic contrast echocardiography (TTCE), also known as bubble 
echocardiography, is the first line management to screen for PAVMs. TTCE has a very high sensitivity (97\%) but is limited in its specificity (49-77\%). If TTCE is not available at a particular centre, chest CT is considered a reasonable alternative for screening. If a patient is not found to have any PAVMs, screening should be completed every 5-10 years. ${ }^{7,10}$

Two thirds of cases of HHT involving neurologic symptoms are caused by pulmonary arteriovenous malformations. In the remaining third, cerebral or spinal arteriovenous malformations cause subarachnoid hemorrhage, or seizure. ${ }^{4}$ Lesions in the brain can be treated with microsurgical excision, stereotactic radiotherapy or embolization. A few studies have shown patients who underwent microsurgery achieved superior obliteration of the lesion and less immediate mortality when compared to stereotactic radiotherapy. ${ }^{8}$ Screening for CVMs is done with MRI and should be completed at the time of diagnosis regardless of age. If a CVM is not identified at diagnosis, no further screening is needed with the exception of the individual diagnosed under the age of 18 . In this case, the patient should be screed once as an adult to ensure no new CVMs have formed. ${ }^{10}$

As previously mentioned, the most common symptom of HHT is epistaxis. This can present with a wide range of severity. Chronic epistaxis has a profound impact on a patient's quality of life and can be life threatening in some cases. ${ }^{11}$ The goal in treating epistaxis caused by HHT is to reduce the frequency and severity of bleeding, reduce the number of transfusions needed, and to improve patient quality of life. There is no standard treatment for HHT epistaxis and the modes of treatment are varied including: nasal packing, electric and/or chemical cauterization, vessel ligation, dermoseptoplasty, topical estrogen, hormonal therapy, and laser treatment. None of these treatments have complete success and often multiple therapies are needed for optimal outcomes. Treatment is determined by the severity of the bleeding and availability of treatment modalities. ${ }^{12}$ Recently, thalidomide has been proposed for the treatment of severe HHT epistaxis with promising preliminary results. ${ }^{13}$

The prognosis of individuals with HHT is generally good, with the majority of patients living a normal life expectancy. Approximately 10\% of HHT patients will die from complications due to their disease with the complications typically involving the central nervous system. ${ }^{8}$

\section{Conclusion}

Hereditary hemorrhagic telangiectasia is a multi systemic disease with the most severe complications arising from bleeding and arteriovenous malformations in the central nervous system, lungs, digestive system and nose. The typical patient suffers from chronic epistaxis and has telangiectasia on fingers, lower lip, buccal mucosa, and tong. Early detection is important and the Curaçao criteria should be used to aid in the clinical diagnosis and to trigger investigations for milder forms of the disease in the patient's family. Due to its multi-systemic manifestations, long-term management of HHT should be completed by a multidisciplinary team and by physicians with experience managing HHT. Treatment is focused primarily on reduction of blood loss and is dependent on symptom severity. Further research is needed to identify optimal treatment algorithms and standardize patient care.

\section{References}

1. Berg J, Gallione C, Stenzel T, Johnson D, Allen W, Schwartz C. et al. The activin receptor-like kinase 1 gene: genomic structure and mutations in hereditary hemorrhagic telangiectasia type 2 . Am J Hum Genet 1997;61(1):60-7.

2. Govani FS, Shovlin C. Hereditary haemorragic telangiectasia: a clinical and scientific review. Eur J Hum Genet 2009;17(7):860-71.

3. McAllister K, Grogg K, Johnson D, Gallione C, Baldwin P, Jackson C. et al. Endoglin, a TGF-beta binding protein of endothelial cells, is the gene for hereditary haemorrhagic telangiectasia type 1 . Nat Genet 1994;8(4):345-51.

4. Guttmacher AE, Marchuk DA, White RI Jr. Hereditary hemorrhagic telangiectasia. N Engl J Med 1995;333(14):918-24.

5. van Gent M, Velthuis S, Post M, Snijder R, Westermann C, Letteboer $\mathrm{T}$, et al. Hereditary hemorrhagic telangiectasia: how accurate are the clinical criteria? Am J Med Genet A 2013;161A(3):461-6.

6. Pierucci P, Lenato M, Suppressa P, Lastella P, Triggiani V, Valerio $\mathrm{R}$, et al. A long diagnostic delay in patients with hereditary haemorrhagic telangiectasia: a questionnaire-based retrospective study. Orphanet J Rare Dis 2012;7:33.

7. Garg N, Khunger M, Gupta A, Kumar N. Optimal management of hereditary hemorrhagic telangiectasia. J Blood Med 2014;5:191206.

8. Shovlin CL, Letarte M. Hereditary haemorrhagic telangiectasia and pulmonary arteriovenous malformations: issues in clinical management and review of pathogenic mechanisms. Thorax 1999;54(8):714-29.

9. van Gent MW, Post MC, Snijder RJ, Westermann CJ, Plokker HW, Mager JJ. Real prevalence of pulmonary right-to-left shunt according to genotype in patients with hereditary hemorrhagic telangiectasia: a transthoracic contrast echocardiography study. Chest 2010;138(4):833-9.

10. Faughnan M, Palda V, Garcia-Tsao G, Geisthoff U, McDonald J, Proctor D, et al. International guidelines for the diagnosis and management of hereditary haemorrhagic telangiectasia. J Med Genet 2011;48(2):73-87.

11. Shin SH, Chae HS, Ji JS, Kim HK, Cho YS, Chang ED, et al. A case of blue rubber bleb nevus syndrome. Korean J Intern Med 2008;23(4):208-12.

12. Juares A, Dell'Aringa A, Nardi J, Kobari K, Rodrigues V, Perches Filho R. Rendu-Osler-Weber Syndrome: case report and literature review. Braz J Otorhinolaryngol 2008;74(3):452-7.

13. Franchini M, Frattini F, Crestani S, \& Bonfanti C. (2013). Novel treatments for epistaxis in hereditary hemorrhagic telangiectasia: a systematic review of the clinical experience with thalidomide. J Thromb Thrombolysis 2013;36(3):355-7. 